\title{
A Note on "Existence Results for Noncoercive Mixed Variational Inequalities in Finite Dimensional Spaces"
}

\author{
Alfredo lusem ${ }^{1} \cdot$ Felipe Lara ${ }^{2}$ \\ Published online: 30 July 2020 \\ (๑) Springer Science+Business Media, LLC, part of Springer Nature 2020
}

\section{Abstract}

We correct the proofs of a previous publication.

Keywords Asymptotic analysis - Asymptotic functions - Noncoercive Optimization · Variational Inequalities $\cdot$ Equilibrium Problems

Mathematics Subject Classification 90C25 $\cdot 90 \mathrm{C} 26 \cdot 90 \mathrm{C} 30$

\section{Introduction}

We correct the proofs of [1, Corollary 3.1 and Theorem 3.2].

\section{The Corrected Proofs}

In the proof of [1, Corollary 3.1], we say "Since assumption (Th) holds immediately for $T(x)=A x+a$ ". This is not correct, as shown in the example given in the paper itself [1, page 127]. So, given a matrix $A \in \mathbb{R}^{n \times n}$, a vector $a \in \mathbb{R}^{n}$, and a closed and convex set $K \subset \mathbb{R}^{n}$, we consider the following assumption:

$(A h)$ : The pair $(A, h)$ has the MVIP on $K$, with MVIP as defined in [1, Definition 3.1].

Hence, [1, Corollary 3.1] should be rewritten as follows:

$\bowtie \quad$ Felipe Lara

felipelaraobreque@gmail.com; flarao@uta.cl

Alfredo Iusem

iusp@impa.br

1 Instituto Nacional de Matemática Pura e Aplicada (IMPA), Rio de Janeiro, Brazil

2 Departamento de Matemática, Facultad de Ciencias, Universidad de Tarapacá, Arica, Chile 
Corollary 2.1 Let A be a $K$-copositive matrix and $a \in \mathbb{R}^{n}$ such that assumption (Ah) holds. If there exists $x_{0} \in K$ such that

$$
h^{\infty}(u)+\left\langle a-A^{\top} x_{0}, u\right\rangle>0, \quad \forall u \in K^{\infty} \backslash\{0\},
$$

then $S(A ; h ; K)$ is nonempty and compact.

In its proof, we replace "Since assumption (Th) holds immediately for $T(x)=$ $A x+a$ " by "By assumption (Ah), assumption (Th) holds for $T(x)=A x+a$ ", and the proof follows.

Analogously, since the proof of [1, Theorem 3.2] is based on [1, Corollary 3.1], we rewrite this theorem as follows:

Theorem 2.1 Let $h: \mathbb{R}^{n} \rightarrow \mathbb{R}$ be a function, and $K$ a nonempty, closed and convex set from $\mathbb{R}^{n}$. Suppose that assumptions $(A 0),(h 0)$ and $(A h)$ hold. Then,

$h^{\infty}(u)+\langle a, u\rangle>0, \quad \forall u \in\left(K^{\infty} \cap \operatorname{Ker} A\right) \backslash\{0\} \Longrightarrow S(A ; h ; K) \neq \emptyset$ and compact.

Finally, in the remainder of the paper, whenever [1, Theorem 3.2] is used, assumption $(A h)$ should be added.

\section{Conclusions}

We have corrected the proofs of a published paper.

Acknowledgements This research was partially supported by Conicyt-Chile throughout Fondecyt Iniciación 11180320 (F. Lara).

\section{Reference}

1. Iusem, A., Lara, F.: Existence results for noncoercive mixed variational inequalities in finite dimensional spaces. J. Optim. Theory Appl. 183, 122-138 (2019)

Publisher's Note Springer Nature remains neutral with regard to jurisdictional claims in published maps and institutional affiliations. 